\title{
Are Evolutionary Debunking Arguments Really Self-Defeating?
}

\author{
Fabio Sterpetti \\ Sapienza University of Rome \\ fabio.sterpetti@uniroma1.it
}

\begin{abstract}
Evolutionary Debunking Arguments (EDAs) are defined as arguments that appeal to the evolutionary genealogy of our beliefs to undermine their justification. Recently, Helen De Cruz and her coauthors supported the view that EDAs are selfdefeating: if EDAs claim that human arguments are not justified, because the evolutionary origin of the beliefs which figure in such arguments undermines those beliefs, and EDAs themselves are human arguments, then EDAs are not justified, and we should not accept their conclusions about the fact that human arguments are unjustified. De Cruz's objection to EDAs is similar to the objection raised by Reuben Hersh against the claim that, since by Gödel's second incompleteness theorem the purpose of mathematical logic to give a secure foundation for mathematics cannot be achieved, mathematics cannot be said to be absolutely certain. The response given by Carlo Cellucci to Hersh's objection shows that the claim that by Gödel's results mathematics cannot be said to be absolutely certain is not self-defeating, and can be adopted to show that EDAs are not selfdefeating as well in a twofold sense: an argument analogous to Cellucci's one may be developed to face De Cruz's objection, and such argument may be further refined incorporating Cellucci's response itself in it, to make it stronger. This paper aims at showing that the accusation of being self-defeating moved against EDAs is inadequate by elaborating an argument which can be considered an EDA and which can also be shown not to be self-defeating.
\end{abstract}

Keywords: evolutionary debunking arguments; scientific realism; self-defeating objection; reduction to the impossible; truth; philosophy of mathematics 


\section{Abbreviations}

EDAs

Vs-MAT

MAT

Vs-EDAs

Pro-EDAs

EDAM

Pro-EDAs+EDAM

EAM

EDA*
Evolutionary Debunking Arguments

Hersh's Objection

Cellucci's Response to Vs-MAT

De Cruz's Objection to EDAs

Response to Vs-EDAs

Evolutionary Debunking Argument for Mathematics

Extended Response to Vs-EDAs

Evolutionary Argument for Mathematics

Simplified Version of Pro-EDAs+EDAM

\section{Introduction}

Evolutionary Debunking Arguments (EDAs) are defined as arguments that appeal to the evolutionary genealogy of our beliefs to undermine their justification (Kahane 2011). The basic structure of EDAs may be described as follows:

[EDAs] (1) We believe that $p$ because we have an intuition that $p$, and there is an evolutionary explanation of our intuition that $p$.

(2) Evolution is not a truth-tracking process.

(3) We are not justified in believing that $p{ }^{1}$

Recently, Helen De Cruz and her co-authors supported the view that EDAs are selfdefeating: if EDAs claim that human arguments are not justified, because the evolutionary origin of the beliefs which figure in such arguments undermines those beliefs, and EDAs themselves are human arguments, then EDAs are not justified, and we should not accept their conclusions about the fact that human arguments are unjustified (De Cruz et al. 2011).

De Cruz's objection to EDAs can also be stated as follows: if human knowledge, as EDAs maintain, is not reliable because human reasoning is not truth-tracking, and human reasoning is not truth-tracking because it is an evolved feature, and evolution by natural

1 Adapted from Kahane 2011, p. 106. 
selection is not a truth aiming process, then even evolutionary theory, which is a product of human reasoning, is not reliable; but "EDAs themselves are based on scientific theories, notably evolutionary theory, and philosophical reflection" (Ibidem, p. 525), then EDAs themselves are not reliable, nor truth-tracking, and so are self-defeating. ${ }^{2}$

It is appropriate to specify that the aim of this paper is limited at assessing whether the self-defeating objection to EDAs obtains or not. The fact is that such objection is often used to support a sort of evolutionary justification of scientific realism (see below). It should be clear that criticizing such objection does not amount to move a general criticism to scientific realism, it is just intended to underline that the proposed evolutionary justification of scientific realism may be unsatisfying, more precisely that one of the arguments used to support such a justification may be unsatisfying.

This paper aims at showing that the accusation of being self-defeating moved against EDAs is inadequate by elaborating an argument which can be considered an EDA and which can also be shown not to be self-defeating. To achieve this goal, we will firstly clarify why the self-defeating objection is worth of attention. Then, we will briefly present Hersh's objection to the claim that mathematical logic cannot give a secure foundation for mathematics, and Cellucci's response to Hersh's objection. Since Hersh's objection is similar to De Cruz's objection against EDAs, we will try to use Cellucci's response to Hersh's objection in order to construct a response to De Cruz's objection. In elaborating such response, we will justify some of the claims attributed to De Cruz's

2 The relevance of evolutionism for epistemology is a well known and debated issue. On that sort of 'Darwinian epistemological dilemma' which arises when we try to naturalize knowledge relying on evolutionism, c.f., e.g., Clark 1986, p. 151: "What is the proper attitude of the evolutionary epistemologist towards science? Should he regard science as disclosing (or aiming to disclose) information concerning the way the world is in itself, independently of the speciesspecific needs, bias and cognitive orientation of the human life-form? Or should he conceive it as intrinsically limited and indelibly marked with the stamp of his own humanity? Either way there is a problem. If he adopts the first, objectivist, interpretation he faces the charge of hypocrisy; why does he not extend the results of his conjectures concerning cognition in other species to the enquiring animal, man? To make that extension, and to regard our scientific knowledge as biased and limited in ways analogous to those attributed to the lower animals, is, however, to breed a deeper discomfort. For if he adopts a species-specific, non-objectivist account of scientific knowledge then the status of the evolutionary conjecture itself is brought into question." 
position, in order to appraise whether this position is fairly described or not. We will then proceed in further developing and making more articulated our proposed response to the self-defeating objection, and we will make more explicit the structure of the argument to better show that it is not self-defeating. Finally, we will make some considerations on some interesting features of the proposed argument, and we will face the objection that this argument is not a genuine evolutionary argument.

\section{The Relevance of the Self-Defeating Objection}

The relevance of the self-defeating objection to EDAs is due to the context in which it has been developed and to the consequences that assessing whether it holds or not may have. In fact, this objection has been raised basically to defend those attempts devoted to develop an evolutionary justification of scientific realism. Indeed, some realists, call them the evolutionary realists, have proposed "that our survival as a species provides some sort of justification for scientific realism" (Thomson 1995, p. 166).

Stich has sketched this position as follows: "the argument seems to be that natural selection favors true beliefs, [...]. So if an organism is the product of natural selection, we can safely assume that most of its beliefs will be true" (Stich 2011, p. 83). In a nutshell, the problem of this position is that if we commit ourselves to claim that human cognitive structures are true beliefs producers because they have been selected for, then we should be able to demonstrate that our cognitive adaptations are able to produce nothing else than (or at least mostly) true beliefs. But this is not an easy task, because, as Stich, among others, has stressed, "it is simply not the case that natural selection favors true beliefs over false ones. What natural selection does favor is beliefs which yield selective advantage. And there are many environmental circumstances in which false beliefs will be more useful than true ones" (Ibidem).

It is not relevant here to give an account of this debate, what is important to underline is that the evolutionary realists have not yet found a fully satisfying defence of their position. ${ }^{3}$ So, to face their opponents, they have elaborated the self-defeating objection. In other words, since their strategy aimed at directly showing that fitness is deeply related to

3 On the difficulties of defending this kind of evolutionary reliabilism, see Sage 2004. For an introduction to the debate on the relation between fitness and truth, see De Cruz et al. 2011. 
the truth seems still controversial, the evolutionary realists argue that denying such claim would lead to an inconsistent position. Thus, whether the self-defeating objection holds or not is relevant to assess the evolutionary realist position.

\section{Hersh's Objection}

Let's turn to the issue at stake, i.e. the above presented De Cruz's objection to EDAs. It is worth noting that De Cruz's objection is similar to the objection raised by Reuben Hersh against the claim that, since by Gödel's second incompleteness theorem the purpose of mathematical logic to give a secure foundation for mathematics cannot be achieved, mathematics cannot be said to be absolutely certain (Cellucci 2013). ${ }^{4}$

Indeed, many authors thought that the purpose of mathematical logic was to give a secure foundation for mathematics and show that mathematics is true and absolutely certain. Following Hilbert, they thought that the only way to prove this claim was to use some absolutely reliable tool, and that the only available absolutely reliable tool was finitary mathematics. In fact, "finitary mathematics is the concrete part of mathematics, which is based on Kant's pure sensible intuition. Infinitary mathematics also involves abstract and problematic concepts, such as that of actual infinity" (Ibidem, p. 220). Being based on Kant's pure sensible intuition, finitary mathematics is considered to be absolutely reliable. "So, if a statement of finitary mathematics is demonstrable in finitary mathematics, this will guarantee that it is true. On the contrary, if a statement of finitary mathematics is demonstrable in infinitary mathematics, this will not guarantee that it is true, because its demonstration may use abstract concepts, which are problematic" (Ibidem).

But this view conflicts with “Gödel's second incompleteness theorem, by which, for any consistent, sufficiently strong, deductive theory $T$, the sentence canonically expressing the consistency of $T, \operatorname{Con}(T)$, is undemonstrable in $T$. Then a fortiori $\operatorname{Con}(T)$ is undemonstrable by absolutely reliable means. Therefore, the purpose of mathematical

\footnotetext{
4 Cellucci specifies that this objection "was raised in correspondence by Reuben Hersh, acting as advocatus diaboli, not because he shared it" (Cellucci 2013, p. 7, footnote 44). So, it is just for simplicity that in what follows we will refer to that objection as 'Hersh's objection'.
} 
logic to give a secure foundation for mathematics cannot be achieved" (Ibidem, p. 4). Thus, mathematics cannot be said to be absolutely certain and true. ${ }^{5}$

Hersh's objection (let's call it: [Vs-MAT]) to this line of reasoning runs as follows:

[Vs-MAT] "If mathematics cannot be said to be absolutely certain, then Gödel's second incompleteness theorem, being a mathematical result, cannot be said to be absolutely certain. But the claim that mathematics cannot be said to be absolutely certain is based on Gödel's result. Then this claim too cannot be said to be absolutely certain. Therefore, the claim that, by Gödel's second incompleteness theorem, mathematics cannot be said to be absolutely certain, is self-defeating" (Ibidem, p. 7).

\section{Cellucci's Response}

The response given by Carlo Cellucci to Hersh's objection shows that the claim that by Gödel's results mathematics cannot be said to be absolutely certain is not self-defeating, and can be adopted to show that EDAs are not self-defeating as well in a twofold sense: an argument with a structure analogous to Cellucci's one may be developed to face De Cruz's objection against EDAs, and such argument may be further developed to incorporate Cellucci's response itself in it, in order to make its defence of EDAs stronger. In fact, as stated above, the aim of this paper is facing the accusation of being selfdefeating moved against EDAs, by elaborating an argument which is an EDA but which

5 This reference to the truth is due to the fact that the truth of mathematics is considered to be related to the certainty of its results, which is given by the reliability of the means used to reach such results, and by their coherence, given that there is no possibility of appealing to some form of empirical confirmation of the truth of a theory when dealing with mathematics. Indeed, Hilbert considered coherence as equivalent to truth: "If the arbitrarily given axioms do not contradict one another, then they are true, and the things defined by the axioms exist. This for me is the criterion of truth and existence" (Hilbert 1980, p. 42). Thus, if by Gödel's results there cannot be absolute certainty in proving the coherence of a mathematical theory, then such theory cannot be said to be absolutely true. 
is not a self-defeating argument. Using Cellucci's response in this twofold sense will be the key to achieve such goal.

To face Hersh's objection, Cellucci has argued that: "this objection is unjustified, because the argument that, by Gödel's second incompleteness theorem, mathematics cannot be said to be absolutely certain, does not depend on the assumption that Gödel's second incompleteness theorem can be said to be absolutely certain" (Ibidem, p. 8). It is a reduction to the impossible (let's call it: [MAT]), which can be described as follows:

[MAT] "Let us suppose, for argument's sake, that mathematics can be said to be absolutely certain. Then Gödel's second incompleteness theorem, being a mathematical result, can be said to be absolutely certain. But, by Gödel's second incompleteness theorem, mathematics cannot be said to be absolutely certain. Thus mathematics cannot be said to be absolutely certain. Contradiction. Therefore mathematics cannot be said to be absolutely certain" (Ibidem).

In order to better develop the analogy between De Cruz's and Hersh's objections and construct a response to De Cruz's objection similar to that given by Cellucci to Hersh, it is possible to reformulate De Cruz's objection (let's call it: [Vs-EDAs]) as follows:

[Vs-EDAs] If human scientific reasoning cannot be said to be truth-tracking, then EDAs, being based on human scientific reasoning, cannot be said to be truth-tracking. But the claim that human scientific reasoning cannot be said to be truth-tracking is based on EDAs. Then this claim too cannot be said to be truth-tracking. Therefore, the claim that, by EDAs, human scientific reasoning cannot be said to be truth-tracking, is self-defeating.

\section{A First Response to De Cruz's Objection}

It is now possible to propose a first Cellucci-style response to De Cruz's objection, i.e. [Vs-EDAs], and claim that this objection is unjustified, because the argument that, by 
EDAs, human scientific reasoning cannot be said to be truth-tracking, does not depend on the assumption that EDAs can be said to be truth-tracking. It is indeed possible to construct such an argument as a reduction to the impossible (let's call it: [Pro-EDAs]), which runs as follows:

[Pro-EDAs] Let us suppose, for argument's sake, that scientific reasoning can be said to be truth-tracking. Then evolutionary theory can be said to be truth-tracking. Then EDAs, being based on evolutionary theory, can be said to be truth-tracking. But, by EDAs, human scientific reasoning cannot be said to be truth-tracking. Thus evolutionary theory cannot be said to be truth-tracking. Contradiction. Therefore human scientific reasoning cannot be said to be truth-tracking. So EDAs are not self-defeating.

This line of reasoning may be articulated by constructing an argument which aims at including the justification of the key-claim of [Pro-EDAs], i.e. that by EDAs human scientific reasoning cannot be said to be truth-tracking, and which incorporates also a reference to the argument, i.e. [MAT], whose structure has been used to produce it. The argument (let's call it: [EDAM]) runs as follows:

[EDAM] Let us suppose, for argument's sake, that our scientific reasoning can be said to be truth-tracking. Then our scientific knowledge, being truth-tracking and reliable, can be said to be true and certain. Thus evolutionary theory, being a scientific theory, can be said to be true and certain.

Thus, if we take that: 1) mathematics derives from our evolved cognitive abilities, which are truth-tracking, and 2) mathematics is indispensable for science, which is true and certain, we can state that mathematics is truth-tracking and certain. Thus, Gödel's incompleteness theorems, being mathematical results, are true and certain. But, by Gödel's second incompleteness theorem, it is impossible to demonstrate by any absolutely reliable means that the axioms we are using are true, and hence that so are the 


\section{PENULTIMATE DRAFT - PLEASE CITE THE PUBLISHED VERSION \\ Philosophia. DOI: 10.1007/s11406-015-9608-4}

theorems proved from them. Thus mathematics cannot be said to be true and certain.

Thus science, if mathematics is indispensable for its being true, and mathematics is not true and certain, cannot be said to be true and certain. But if science is not true and certain, then our scientific reasoning cannot be said to be truth-tracking. Contradiction. Therefore our scientific reasoning cannot be said to be truthtracking.

The crucial claim in the second part of [EDAM], i.e. that mathematics cannot be said to be true and certain, makes an implicit reference to [MAT], and can be justified using this very argument.

\section{Justifying Two Assumptions}

But there are some other claims which appear in the first part of [EDAM] that should be analysed. Are the claims 1) and 2) used in [EDAM] justifiable? Better, is it fair to attribute those claims to De Cruz's position?

Let's start from the first claim: 1) mathematics derives from our evolved cognitive abilities, which are truth-tracking. It has to be kept in mind that De Cruz maintains a sort of evolutionary scientific realism. In fact, she claims that "the brain has as proper function the production of beliefs that are fitness-enhancing," and supports an evolutionary argument, which "contends that natural selection will form animal brains that tend to produce true beliefs, because true beliefs are essential for adaptive decision making" (De Cruz, De Smedt 2012, pp. 416-417). Thus, truth is essential for the survival and to produce beliefs that are fitness-enhancing means to produce true beliefs.

With regard to mathematics, De Cruz explains the applicability of mathematics as follows: since mathematics is the product of evolution by natural selection, it "must somehow have promoted the survival and reproductive success of the ancestors of those organisms. This is only likely if there is some correspondence between those innate cognitive domains, like numerical competence, and the physical world" (De Cruz 2004, pp. 80-81). If the beliefs produced by humans have to be true in order to be fitness- 
enhancing, and mathematics is produced by humans because it has been fitnessenhancing, we can conclude that she thinks that mathematics has to be true.

Let's now turn to the second claim: 2) mathematics is indispensable for science, which is true and certain. From a perspective like that of De Cruz, there is a deep relation between the evolved structure of the human brain and scientific knowledge. In fact, "from an evolutionary perspective, science is a recent development in our species. Thus, scientists have to draw on the same cognitive resources as other people, and they are subject to the same cognitive limitations" (De Cruz 2011, p. 205). There is a continuity between our cognitive structures and science, and since the formers, as stated above, are truth-tracking, also the latter is truth-tracking. But science can go even further and overcome some of our biases and limitations. For example, mathematics is something more than "a reliable tool to describe scientific phenomena" (De Cruz 2004, p. 81). Indeed, mathematical "symbols [...] enable us to represent concepts that are literally unthinkable with our bare brains" (De Cruz, De Smedt 2013, p. 3). Thus, using "mathematical symbols can be" considered "not unlike the use of other external tools in scientific practice, such as microscopes, particle accelerators and slide rulers" (Ibidem, p. 17). Those instruments allow us to overcome some of our biases and limitations, they are indispensable to reach certain scientific results. Since mathematics is equated by De Cruz to such instruments, we can fairly conclude that she thinks that mathematics is indispensable for science.

Finally, that science is considered to be true appears very clearly considering the fact that De Cruz raises the self-defeating objection against EDAs. Indeed EDAs are built denying exactly the claim that fitness and truth are related: ${ }^{6}$ if science, or more generally knowledge, is not true or does not aim at truth, then the self-defeating objection falls. In fact, the self-defeating objection underlines that EDAs are based on science, and that if science is not true, then EDAs themselves cannot be true, and thus EDAs cannot claim to be genuine knowledge. But if science and knowledge are considered not to be aimed at truth, then there is no reason to consider EDAs as self-defeating. In fact, if EDAs claim that our scientific knowledge is not true because of its evolutionary roots, and truth is not necessary to have knowledge, then even if EDAs are based on science and science is not

6 Cf. De Cruz et al. 2011, p. 518: “An EDA is constructed by negating at least one of the crucial EA [Evolutionary Argument] premises, in particular about the relative importance of natural selection, and about its truth-tracking ability." 
true, EDAs are not self-defeating, because they have not to be true in order to claim to be genuine knowledge. Thus, given that De Cruz supports the self-defeating objection, it seems fair to conclude that she thinks that science is true and that knowledge has to be true.

To sum up, considering her insistence on the connection between the survival and the truth, her insistence on the continuity between the evolved truth-tracking cognitive structures and science, and her support to the self-defeating objection to EDAs, the ascription of the claims 1) and 2) which figure in [EDAM] to De Cruz's position seems to be fair.

\section{Developing the Response to the Self-Defeating Objection}

After this brief digression aimed at showing the fairness of [EDAM], we can proceed a little bit further along the direction taken with the second step we made in developing an argument to resist the self-defeating objection. Indeed, the arguments presented above, i.e. [Pro-EDAs] and [EDAM], may be nested to build a single argument (let's call it: [Pro-EDAs+EDAM]), in order both to argue for a debunking position and show that such position is not self-defeating in a more detailed fashion, as follows:

[Pro-EDAs+EDAM] To show that EDAs are not self-defeating, let us suppose, for argument's sake, that scientific reasoning can be said to be truthtracking. Then evolutionary theory can be said to be truth-tracking. Then EDAs, being based on evolutionary theory, can be said to be truth-tracking. But, by EDAs, human scientific reasoning cannot be said to be truth-tracking.

In fact, let us suppose, for argument's sake, that our scientific reasoning can be said to be truth-tracking. Then our scientific knowledge, being truth-tracking and reliable, can be said to be true and certain. Thus evolutionary theory, being a scientific theory, can be said to be true and certain. Thus, if we take that: 1) mathematics derives from our evolved cognitive abilities, which are truthtracking, and 2) mathematics is indispensable for science, which is true and certain, we can state that mathematics is truth-tracking and 
certain. Thus, Gödel's incompleteness theorems, being mathematical results, are true and certain. But, by Gödel's second incompleteness theorem, it is impossible to demonstrate by any absolutely reliable means that the axioms we are using are true, and hence that so are the theorems proved from them. Thus mathematics cannot be said to be true and certain. Thus science, if mathematics is indispensable for its being true, and mathematics is not true and certain, cannot be said to be true and certain. But if science is not true and certain, then our scientific reasoning cannot be said to be truth-tracking. Contradiction. Therefore our scientific reasoning cannot be said to be truth-tracking.

Thus evolutionary theory cannot be said to be truth-tracking. Contradiction. Therefore human scientific reasoning cannot be said to be truth-tracking. So EDAs are not self-defeating.

In order to better analyse this last argument, i.e. [Pro-EDAs+EDAM], and the relation between its constituent parts, it is possible to sketch a simplified version of the different arguments put together to form it and the specular arguments which oppose to them. They may be grouped in three couples: (1) the arguments which argue whether Cellucci's claim on mathematics is self-defeating or not; (2) the arguments which argue whether EDAs are self-defeating or not; (3) the arguments which argue whether a naturalistic view of mathematics implies its being true or not. It can easily be noted that [Pro-EDAs+EDAM] is made up by putting together (1b), (2b) and (3b), and that none of them is selfdefeating, they are just reductions to the impossible:

(1a) [Vs-MAT] Mathematics cannot be said to be true. Then Gödel's second incompleteness theorem, being a mathematical result, cannot be said to be true. But the claim that mathematics cannot be said to be true is based on Gödel's result. Then this claim too cannot be said to be true. Therefore, the claim that, by Gödel's second incompleteness theorem, mathematics cannot be said to be true, is self-defeating. 
(1b) [MAT] Mathematics can be said to be true. Then Gödel's second incompleteness theorem, being a mathematical result, can be said to be true. But, by Gödel's second incompleteness theorem, it is impossible to demonstrate by any absolutely reliable means that the axioms we are using are true, and hence that so are the theorems proved from them. Thus mathematics cannot be said to be true. Contradiction. Therefore mathematics cannot be said to be true.

(2a) [Vs-EDAs] Human scientific reasoning cannot be said to be truth-tracking. Then EDAs, being based on human scientific reasoning, cannot be said to be truth-tracking. But the claim that human scientific reasoning cannot be said to be truth-tracking is based on EDAs. Then this claim too cannot be said to be truth-tracking. Therefore, the claim that, by EDAs, human scientific reasoning cannot be said to be truth-tracking, is self-defeating.

(2b) [Pro-EDAs] Scientific reasoning can be said to be truth-tracking. Then evolutionary theory can be said to be truth-tracking. Then EDAs, being based on evolutionary theory, can be said to be truthtracking. But, by EDAs, human scientific reasoning cannot be said to be truth-tracking. Thus evolutionary theory cannot be said to be truth-tracking. Contradiction. Therefore human scientific reasoning cannot be said to be truth-tracking. So EDAs are not self-defeating.

(3a) $[\mathrm{EAM}] \quad$ Mathematics is due to our evolved cognitive abilities. Our evolved cognitive abilities are truth-tracking. Then mathematics can be said to be truth-tracking. Then mathematics can be said to be true.

(3b) [EDAM] Mathematics is due to our evolved cognitive abilities. Our evolved cognitive abilities are truth-tracking. Then mathematics can be said to be truth-tracking. Then mathematics can be said to be true. But, by $[\mathrm{MAT}]$ mathematics cannot be said to be true. Thus 
mathematics cannot be said to be truth-tracking. Contradiction. Therefore our evolved cognitive abilities cannot be said to be truthtracking.

To let the structure of [Pro-EDAs+EDAM] be more clear, and make visually explicit the role that [MAT], i.e. the argument from which we moved to develop a response to the self-defeating objection, plays in it, and the way in which the arguments by which it is composed are nested, using (1) and (2) to designate the first and the second part of an argument which includes another argument, and using 'a', 'b', 'c' to designate the main claims of the argument, [Pro-EDAs+EDAM] may be sketched as follows:

[Pro-EDAs] (1) Suppose that scientific reasoning can be said to be truth-tracking. Then evolutionary theory can be said to be truth-tracking [a]. Then EDAs, being based on evolutionary theory, can be said to be truthtracking. But, by EDAs, human scientific reasoning cannot be said to be truth-tracking.

[EDAM] (1) In fact, the following argument may be developed: Mathematics is due to our evolved cognitive abilities. Suppose that our evolved cognitive abilities are truth-tracking. Then mathematics can be said to be truth-tracking [b]. Then mathematics can be said to be true. But, by $[\mathrm{MAT}]$ mathematics cannot be said to be true.

[MAT] In fact, the following argument may be developed: Suppose that mathematics can be said to be true [c]. Then Gödel's second incompleteness theorem, being a mathematical result, can be said to be true. But, by Gödel's second incompleteness theorem, it is impossible to demonstrate by any absolutely reliable means that the axioms we are using are true, and hence that so are the theorems proved from them. Thus mathematics cannot be said to be true $(\neg[\mathrm{c}])$. Contradiction. Therefore mathematics cannot be said to be true. 
[EDAM] (2) Thus mathematics cannot be said to be truth-tracking ( $\neg[\mathrm{b}]$ ). Contradiction. Therefore our evolved cognitive abilities cannot be said to be truth-tracking.

[Pro-EDAs] (2) Thus evolutionary theory cannot be said to be truth-tracking ( $\neg[\mathrm{a}])$. Contradiction. Therefore human scientific reasoning cannot be said to be truth-tracking. Therefore EDAs are not self-defeating.

\section{Some Features of the Proposed Response}

It should now be transparent that [Pro-EDAs+EDAM] is made up by giving in (2b), i.e. [Pro-EDAs], as an instantiation of the claim that by EDAs, human scientific reasoning cannot be said to be truth-tracking, [EDAM]. [EDAM] in fact is an EDA made up by the conjunction of (3a), i.e. [EAM], which constitutes [EDAM] first part, and (1b), i.e. [MAT], which constitutes [EDAM] second part.

Thus, if [EAM] and [MAT] are accepted, then [EDAM] follows. If [EDAM] is accepted, then [Pro-EDAs] follows. The fact is that if mathematics is naturalistically intended as being rooted in our evolved cognitive abilities, and evolutionary theory is considered both 1) true; and 2) being able to show that our evolved cognitive abilities are truth-tracking, i.e. if we construct an Evolutionary Argument for Mathematics [EAM]; and if by this argument we are justified in believing that mathematical results are true, then it is a very mathematical result which contradicts such argument.

Thus, an Evolutionary Debunking Argument for Mathematics [EDAM] may be conceived of as a reduction to the impossible of the proposed [EAM]. In other words, $[E D A M]$ is structured in such a way that the more $[E A M]$ is true, and thus mathematics is true, the more the Gödel's results are true and inescapable. And so, the more the truth of such results is justified by [EAM], the more [EDAM] is stronger. This feature of [EDAM] is due to the peculiar role that mathematics plays in science and to the uniqueness of Gödel's results. This is the reason why we focused on Cellucci's Response.

Since $[E A M]$ is exactly the position advocated by De Cruz, [EDAM] puts pressure on such position underlining a sort of dilemma that the supporters of [EAM] have to face. Moreover, given that [EDAM] seems able to coherently claim that our evolved cognitive abilities cannot be said to be truth-tracking, it is an example of how EDAs can make such 
claim without contradicting any of their premises, and so it allows us to support the claim that EDAs are not self-defeating.

\section{Facing an Objection}

It may be objected that $[\mathrm{EDAM}]$ is not really an evolutionary debunking argument, since it is crucially based on the consequences of some mathematical results, more than on some biological findings relative to evolution.

But this objection is inadequate. Indeed, if we recall the basic structure of EDAs reported above:

[EDAs] (1) We believe that $p$ because we have an intuition that $p$, and there is an evolutionary explanation of our intuition that $\mathrm{p}$.

(2) Evolution is not a truth-tracking process.

(3) We are not justified in believing that $p$.

We can see that the crucial epistemic premise is (2), i.e. evolution is not a truthtracking process. [EDAM] is just an argument which tries to justify such premise relying on a reduction to the impossible, which starts giving for granted that evolution is a truthtracking process in order to show that we should not accept such claim because it leads to a contradiction. Thus, [EDAM] just aims at supporting the crucial epistemic premise of EDAs, i.e. that evolution is not a truth-tracking process, using a reduction to the impossible. And [EDAM] seems to succeed in doing this. The fact that [EDAM] does not rest on some positive result of biology to support that crucial epistemic premise, but instead tries to show the absurdity of the consequences of adopting the negation of that epistemic premise, does not mean that [EDAM] is not an evolutionary debunking argument. Indeed, EDAs deny that our beliefs are justified because of their evolutionary origin, and to do this, EDAs have to justify the claim that evolution is not a truth-tracking process. What qualifies EDAs as evolutionary is exactly the claim that evolution does not track the truth, not the reference to some specific biological result to support this claim. ${ }^{7}$ The fact that the justification of such premise has to be derived exclusively from biology

7 See above footnote 6. 
or that it has not to be presented in the form of a reduction to the impossible, does not figure in any characterization of EDAs.

We can reformulate the argument described above, i.e. [Pro-EDAs+EDAM], in a simplified way as [EDA*] to better show that it fits the reported description of EDAs, as follows:

[EDA*] (1) We believe that scientific reasoning is truth-tracking because we have an intuition that scientific reasoning is truth-tracking, ${ }^{8}$ and there is an [evolutionary explanation] of our intuition that scientific reasoning is truth-tracking.

[evolutionary explanation]: science is possible thanks to our evolved cognitive abilities; evolved cognitive abilities are truthtracking because they have been selected by natural selection, and natural selection selects for truth-tracking abilities, because truthtracking abilities are fitness enhancing and natural selection maximizes fitness.

(2) Evolution is not a truth-tracking process.

[justification for (2)]: Evolution is not a truth-tracking process because fitness is not related to the truth, so the fact that some feature has been selected does not guarantee that such feature is truth-tracking (crucial Epistemic Claim of EDAs: EC).

[justification for EC]: To see that fitness is not related to the truth, let us suppose, for argument's sake, that that our evolved cognitive abilities are truth-tracking, etc. (i.e.: [EDAM]).

(3) We are not justified in believing that scientific reasoning is truth-tracking.

8 On the fact that the main idea which lays behind scientific realism is the intuition that the empirical success of a scientific theory can be explained by nothing but its truth, see, e.g., Worrall 1989. 
This formulation makes clear that the proposed response to De Cruz's objection may be fairly considered an EDA.

\section{Conclusions}

To sum up, [EDAM] poses a challenge to those who want to support the claim that an evolutionary view of mathematics justifies its truth and that a scientific realist stance grounded on evolutionism may be compatible with the previous claim, showing a sort of dilemma that such position has to face: the more mathematics is true, the less evolutionary theory may justify the truth of our scientific theories, including evolutionary theory itself. But the justification of the truth of mathematics rests on evolutionary theory. On the other hand, if evolutionary theory is true and natural selection promotes truthtracking abilities, and we claim that natural selection gave us the ability to produce mathematics, then mathematics has to be true. But, as we have seen, the more mathematics is true, the less evolutionary theory may justify the truth of our scientific theories, etc. In moving this challenge, [EDAM] puts under pressure the evolutionary claim that evolution is a truth-tracking process. Since denying such claim is one of the characteristic features of EDAs, it seems fair to say that [EDAM] is a genuine EDA. Thus, the objection according to which [EDAM] is not really an EDA is inadequate.

It seems that we can safely conclude that [Pro-EDAs+EDAM] is an EDA, and that it not a self-defeating argument, and so that it is a good candidate to face the self-defeating objection raised against EDAs.

\section{References}

Cellucci, C. (2013). Rethinking Logic. Logic in Relation to Mathematics, Evolution, and Method. Dordrecht: Springer.

Clark, A.J. (1986). Evolutionary Epistemology and the Scientific Method. Philosophica, $37,151-162$.

De Cruz, H. (2004). Why humans can count large quantities accurately. Philosophica, 74, 63-83.

De Cruz, H. (2011). Through a mind darkly. PhD Thesis, Groningen: University of Groningen. 
De Cruz, H., Boudry, M., De Smedt, J., Blancke, S. (2011). Evolutionary Approaches to Epistemic Justification. Dialectica, 65(4), 517-535.

De Cruz, H., De Smedt, J. (2012). Evolved cognitive biases and the epistemic status of scientific beliefs. Philosophical Studies. 157(3), 411-429.

De Cruz, H., De Smedt, J. (2013). Mathematical symbols as epistemic actions. Synthese, 190(1), 3-19.

Hilbert, D. (1980). Letter to Frege 29.12.1899. In: Frege, G., Philosophical and mathematical correspondence (pp. 41-43). Oxford: Blackwell.

Kahane, G. (2011). Evolutionary Debunking Arguments. Noûs, 45(1), 103-125.

Sage, J. (2004). Truth-Reliability and the Evolution of Human Cognitive Faculties. Philosophical Studies, 117(1-2), 95-106.

Stich, S. (2011). Collected Papers. Volume 1. Oxford: Oxford University Press.

Thomson, P. (1995). Evolutionary Epistemology and Scientific Realism. Journal of Social and Evolutionary Systems, 18(2), 165-191.

Worrall, J. (1989). Structural Realism: The Best of Both Worlds? Dialectica, 43(1-2), 99-124. 